\title{
Geophysical assessments of renewable gas energy compressed in geologic pore storage reservoirs
}

\author{
Said Attia al Hagrey*, Daniel Köhn and Wolfgang Rabbel
}

\begin{abstract}
Renewable energy resources can indisputably minimize the threat of global warming and climate change. However, they are intermittent and need buffer storage to bridge the time-gap between production (off peak) and demand peaks. Based on geologic and geochemical reasons, the North German Basin has a very large capacity for compressed air/gas energy storage CAES in porous saltwater aquifers and salt cavities. Replacing pore reservoir brine with CAES causes changes in physical properties (elastic moduli, density and electrical properties) and justify applications of integrative geophysical methods for monitoring this energy storage. Here we apply techniques of the elastic full waveform inversion FWI, electric resistivity tomography ERT and gravity to map and quantify a gradually saturated gas plume injected in a thin deep saline aquifer within the North German Basin. For this subsurface model scenario we generated different synthetic data sets without and with adding random noise in order to robust the applied techniques for the real field applications. Datasets are inverted by posing different constraints on the initial model. Results reveal principally the capability of the applied integrative geophysical approach to resolve the CAES targets (plume, host reservoir, and cap rock). Constrained inversion models of elastic FWI and ERT are even able to recover well the gradual gas desaturation with depth. The spatial parameters accurately recovered from each technique are applied in the adequate petrophysical equations to yield precise quantifications of gas saturations. Resulting models of gas saturations independently determined from elastic FWI and ERT techniques are in accordance with each other and with the input (true) saturation model. Moreover, the gravity technique show high sensitivity to the mass deficit resulting from the gas storage and can resolve saturations and temporal saturation changes down to $\pm 3 \%$ after reducing any shallow fluctuation such as that of groundwater table.
\end{abstract}

Keywords: Renewable energy; Compressed air/gas energy storage (CAES); Elastic full waveform inversion (FWI); Electric resistivity tomography (ERT); Gravity method; Petrophysical rock parameters

\section{Introduction}

One unprecedented challenge facing the human being is the energy resources, and its coupling with global climate changes and warming from greenhouse gases (GHG). Mitigation of anthropogenic GHG, including $\mathrm{CO}_{2}$ emissions in the terrestrial atmosphere demands developments of viable alternative of renewable energy resources including hydroelectric, biomass, solar, wind, marine (wave/ tides) and geothermal sources. Most of these sources produce energy only when suitable weather conditions are

\footnotetext{
* Correspondence: sattia@geophysik.uni-kiel.de

Department of Geophysics, Institute of Geosciences, University of Kiel, 24118 Kiel, Germany
}

\section{Springer}

prevailing and not when energy directly demanded. These sources are intermittent and need buffer storage to bridge the time-gap (disparity) between off-peak production and demand peaks. The underground geology offers an adequate option for short- and long-term energy storage such as compressed air or gas energy storage, CAES (e.g., Crotogino et al. 2001; Succar and Williams 2008). The North German Basin delivers favourable conditions (geological, geochemical) for underground space utilization and has a huge capacity for CAES in porous brine aquifers and salt caverns (natural and artificial). Advantages of renewable energy storage are (1) balancing power demand and fluctuating renewable energy production, (2) bridging 
temporal mismatch between renewable energy production (off-peaks) and demand (peaks), i.e., storing off-peak energy supply to use it during peak demand periods, and (3) offering large buffer capacity to meet any disruptions in energy supply.

Figure 1 shows how a renewable electricity system could supply actual electricity demand during one week in Minnesota (Makhijani et al. 2012). The daily electricity demand is constantly changing while the surplus renewable generation is put into storage. The basic approach of CAES is as follows: When electricity generation is greater than demand, the energy surplus is used to compress air in the geostorage. When generation is less than demand, pressurized air is withdrawn from storage and used to drive an electricity turbine.

In a geological gas storage in saline formations, the gas replaces the pore brine causing strong changes in elastic moduli, density and electric resistivity. These physical contrasts justify the application of integrative geophysical techniques for monitoring this geostorage. These include techniques of elastic full waveform inversion (FWI), electric resistivity tomography (ERT), gravity and electromagnetic induction (EMI) of time- (TEM) and frequency-domain (FEM). EMI techniques (ground and air based) are applied usually for monitoring shallow targets, e.g., leakages in groundwater.

Since some years ago Germany practices a turnaround in the energy policy (German "Energiewende") and is currently leading in the production of solar and wind energy (IEA 2013). Wind energy are produced mainly at the coastal areas (on-shore and off-shore) of North Germany which is characterized by high wind speeds. We started 2012 an interdisciplinary joint research project ANGUS+ dealing with impacts of using geologic subsurface as a thermal, electric or material storage in context with alternative energy resources (Bauer et al. 2013). This includes dimensioning, risk analyses and impact predictions as a base for future space planning of the subsurface. Our main task is to develop a geophysical monitoring strategy using integrative approach of geophysical techniques (FWI, ERT, EMI and gravity) on almost realistic scenarios in the North German Basin.

We show here results of numerical simulations of elastic FWI, ERT and gravity techniques in mapping CAES reservoirs with a continuous gradual desaturation with depth. These simulations are applied for synthetic data (before and after adding 3\% random noise error) and inverted using constrains on the initial models.

\section{Gas storages in the North German Basin}

For a more realistic modelling scenario we selected a synthetic site in the North German Basin for this numerical study of CAES in geologic formations. The model block of this site $\left(29 * 28 * 5.5 \mathrm{~km}^{3}\right)$ consists of a thick succession of 14 sedimentary layers ranging in age from Permian to Tertiary (Figure 2, Baldschuhn et al. 2001; Hese 2012). The succession shows nearly horizontal layering with a gentle anticline fold in its southern part. It shows an unconformity, where formations of the Lower cretaceous, Lias and Rhaet disappear within the anticline crest at the depth interval of $0.7-1.0 \mathrm{~km}$. The succession includes two thin brine reservoirs of a porous sandstone (5-30 m thickness), namely the Rhaet (1-1.5 km depth) and Quickborn (2-2.5 km depth) formations. Both are potential pore reservoirs for CAES and only the shallow Rhaet formation is considered in this study. The compressed gas is injected in the uppermost formation part within the northern anticline limb. Here this very light gas (e.g., air density of $1.29 \mathrm{~kg} / \mathrm{m}^{3}$ at STP "standard temperature, $0^{\circ} \mathrm{C}$, and pressure, $101325 \mathrm{~Pa}$ ") replaces the dense brine of $>1100 \mathrm{~kg} / \mathrm{m}^{3}$ (with total dissolved solids, TDS, of $>100 \mathrm{~g} / \mathrm{l}$ at this depth) in the pores of the sandstone reservoir. Accordingly, the compressed gas saturation approaches its maximum value directly below the injection level and decreases gradually with depth within the reservoir of the dipping anticline flank. This downward gradual gas desaturation may correspond well with the almost realistic $\mathrm{CO}_{2}$ plume scenario injected

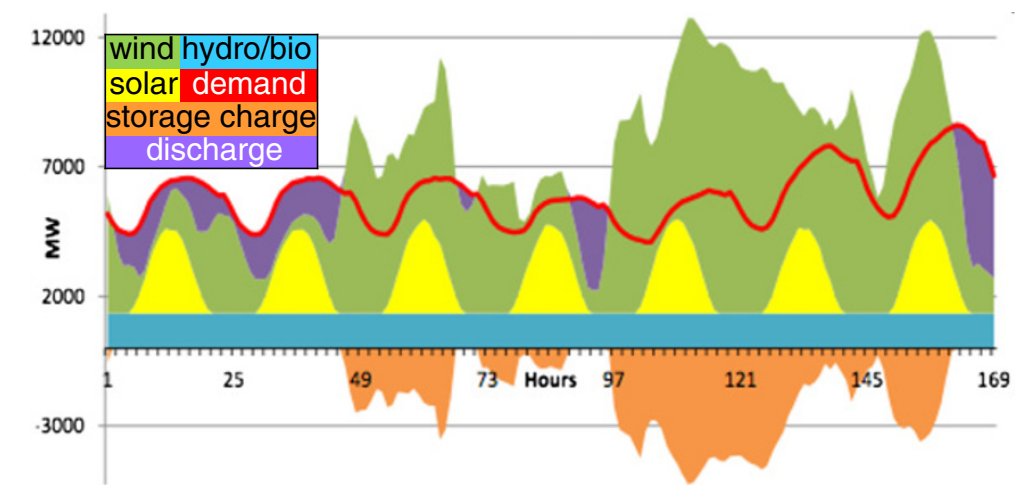

Figure 1 Daily supply and demand with storage of renewable energy, 11-17 July 2007, Minnesota (Makhijani et al. 2012). 

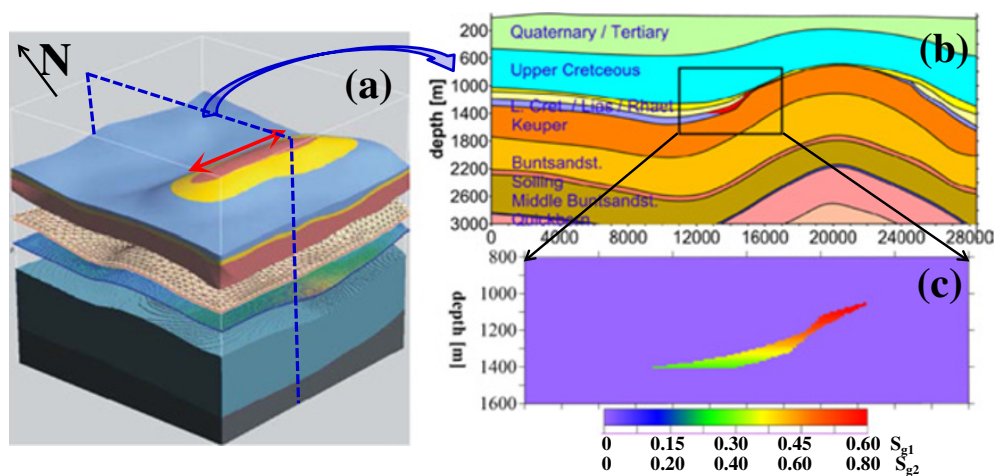

Figure 2 Study site within the North German Basin. (a) 3D block below the Quaternary-Tertiary overburden, (b) 2D stratigraphy section perpendicular to the main anticline structure, and $(\mathbf{c}) 2 \mathrm{D}$ saturations of compressed gas $\left(S_{q 1}\right.$ and $\left.S_{q 1}\right)$ injected in the saline Rhaet reservoir of the northern limb, (partly from Hese 2012).

in a deep saline aquifer (Graupner et al. 2011). A combined simulation of multiphase flow, transport and geochemical reactions in a brine reservoir shows that the gas phase saturation decreases with increasing distance away from the injection point. The limited lateral extension of the thin Rhaet reservoir leads to dominating the downward gas propagation, where the porosity and permeability decrease generally with depth. For this reservoir we applied two saturation distributions $\left(S_{g 1}\right.$ and $\left.S_{g 2}\right)$ gradually decreasing downwards of the range $0.60-0.27$ and $0.80-0.36$, respectively (Figure 2c). The applied gradual desaturation with depth $(z)$ follows this function: $S_{g}=S_{g 0} e^{-5\left(z-z_{0}\right)^{2}}$, with $S_{g 0}=$ maximum $S_{g}$ at $z_{0}=1 \mathrm{~km}, 1 \mathrm{~km}>z \leq 1.4 \mathrm{~km}$. Both of $S_{g 1}$ and $S_{g 2}$ approach their maximum values near to the anticline crest (at $1 \mathrm{~km}$ depth) and gradually decrease with depth according to this function approaching their minimum values at $1.4 \mathrm{~km}$ depth.

\section{The seismic forward problem, FWI and model parameterization}

The propagation of seismic waves in an isotropic elastic medium can be described by a system of coupled first order partial differential equations (Landau and Lifschitz 1986)

$$
\begin{aligned}
& d \frac{\partial v_{i}}{\partial t}=\frac{\partial \sigma_{i j}}{\partial x_{j}}+f_{i} \\
& \frac{\partial \sigma_{i j}}{\partial t}=\lambda\left(\frac{\partial \epsilon_{11}}{\partial t}+\frac{\partial \epsilon_{22}}{\partial t}+\frac{\partial \epsilon_{33}}{\partial t}\right) \delta_{i j}+2 \mu \frac{\partial \epsilon_{i j}}{\partial t}+\frac{\partial T_{i j}}{\partial t} \\
& \frac{\partial \epsilon_{i j}}{\partial t}=\frac{1}{2}\left(\frac{\partial v_{i}}{\partial x_{j}}+\frac{\partial v_{j}}{\partial x_{i}}\right)
\end{aligned}
$$

where $d$ denotes the density, $v_{i}$ the particle velocity, $\sigma_{i j}$ the stress tensor, $\varepsilon_{i j}$ the strain tensor, $\lambda$ and $\mu$ the Lamé parameters, $\delta_{i j}$ the Kronecker Delta, $f_{i}$, $T_{i j}$ the source terms for body and surface forces, respectively.

Equation (1) is a general expression for the conservation of momentum in a continuum. It is independent of the medium state - such as gas, fluid or solid. To describe the behavior of the material correctly a relationship between the forces (stresses $\sigma_{i j}$ ) acting on the medium and the resulting deformation (strain $\varepsilon_{i j}$ ) is required. For small forces/deformations and an isotropic medium this relationship is linear (generalized Hooke's law) depending only on the distibution of two material parameters $\lambda$ and $\mu$ (Lamé parameters). Assuming that these parameters are time-independent the stress-strain relationship can be replaced by the stress-strain-rate equation (2). For a given isotropic elastic medium equations $(1-3)$ can be solved numerically and therefore synthetic seismograms for any acquisition geometry calculated. Based on the solution of the seismic forward problem a high-resolution imaging concept called full waveform inversion (FWI) has been developed in the 1980s by Tarantola (1986). Since then the FWI is significantly improved and applied to a wide range of field applications (Virieux and Operto 2009).

The aim of (spatial) FWI is to minimize the data residuals $\delta u=u^{\text {mod }}-u^{\text {obs }}$ between the modelled data $u^{\text {mod }}$ and the field data $u^{o b s}$ to deduce high resolution models of the elastic material parameters in the underground. To solve this nonlinear optimization problem an appropriate objective function $E$ has to be defined. Similar to Asnaashari et al. (2013a), we use the following objective function

$$
E=\frac{1}{2}\left(\|\boldsymbol{\delta} \boldsymbol{u}\|_{2}+\lambda_{1}\left\|\boldsymbol{W}_{\boldsymbol{m}}\left(\boldsymbol{m}-\boldsymbol{m}_{\text {prior }}\right)\right\|_{2}\right)
$$

Where the term $\frac{1}{2}\|\boldsymbol{\delta} \boldsymbol{u}\|_{2}$ denotes the $\mathrm{L}_{2}$-norm of the data misfit and $\frac{\lambda_{1}}{2}\left\|\boldsymbol{W}_{\boldsymbol{m}}\left(\boldsymbol{m}-\boldsymbol{m}_{\text {prior }}\right)\right\|_{2}$ a weighted $\mathrm{L}_{2}$ norm of the difference between the model parameters $m$ 
and prior model information $m_{\text {prior }}$ used for model regularization. The parameter $\lambda_{1}$ balances the contributions of the data misfit and the model regularization term, while the spatial variable weighting factor $W_{m}$ defines which parts of the model are updated during the inversion process. The spatial weighting of the model updates is crucial for a successful inversion, because near surface inversion artefacts can introduce artificial data residuals not present in the real time-lapse data and therefore lead to an increase of the nonlinearity of the inverse problem. Like Asnaashari et al. (2013b) the magnitudes of the spatial weighting factors are based on elastic reverse-time migration (RTM) results to restrict model updates to the storage formation. The objective function equation (4) can be minimized by iteratively updating the model parameters $\boldsymbol{m}_{n}$ (P-wave velocity $\left(V_{p}\right)$, S-wave velocity $\left(V_{s}\right)$, density $\left.(d)\right)$ at iteration step $\mathrm{n}$, starting with an initial background model $\boldsymbol{m}_{\boldsymbol{o}}$ using the Newton method (Nocedal and Wright 2006):

$$
\boldsymbol{m}_{n+1}=\boldsymbol{m}_{n}-\tau_{n}\left(\boldsymbol{H}_{\boldsymbol{m}}^{-1} \boldsymbol{G}_{\boldsymbol{m}}\right)_{\boldsymbol{n}}
$$

with the gradient of the objective function equation (4) with respect to the elastic model parameters

$$
\boldsymbol{G}_{m}=\frac{1}{2}\left(\frac{\partial\|\boldsymbol{\delta} \boldsymbol{u}\|_{2}}{\partial \boldsymbol{m}}\right)+\boldsymbol{\lambda}_{1} \boldsymbol{W}_{\boldsymbol{m}}{ }^{2}\left(\boldsymbol{m}-\boldsymbol{m}_{\text {prior }}\right)
$$

and $H_{m}$ the second derivative of the objective function (Hessian). An explicit calculation of the Hessian in the time-domain is computational very expensive. Therefore, we use the quasi-Newton L-BFGS (Limited-memory Broyden-Fletcher-Goldfarb-Shanno) technique (Nocedal and Wright 2006; Brossier 2011), where the product of the inverse Hessian $H_{m}{ }^{-1}$ with the gradient $G_{m}$ is iteratively approximated by finite-differences.

The effective calculation of the time-domain gradient directions $\left(\frac{\partial\|\boldsymbol{\delta} \boldsymbol{u}\|_{2}}{\partial \boldsymbol{m}}\right)$ with the adjoint method for different model parameterizations are described in Tarantola (1986), Mora (1987), Shipp and Singh (2002) and Köhn et al. (2012). The step length $\tau_{\mathrm{n}}$ is estimated by a linesearch satisfying the Wolfe-conditions (Nocedal and Wright 2006) to assure a fast and accurate convergence of the L-BFGS algorithm. While Asnaashari et al. (2013a) introduce another regularization term to assure model smoothness, we apply a weak wavenumber domain filter to the estimated search directions at every iteration step for the same purpose.

In elastic time-lapse FWI, the data residuals are modified according to

$$
\boldsymbol{\delta} \mathbf{u}=\left(\left\{\boldsymbol{u}^{\text {mod }}\left(t_{1}\right)-\boldsymbol{u}^{\text {mod }}\left(t_{0}\right)\right\}-\left\{\boldsymbol{u}^{\text {obs }}\left(t_{1}\right)-\boldsymbol{u}^{\boldsymbol{o b s}}\left(t_{0}\right)\right\}\right),
$$

which denote the difference between the modelled and the field data at time steps $t_{0}$ (baseline model) and $t_{1}$
(Denli and Huang 2009). This redefinition of the data residuals leads to a much stronger focusing of the model updates at reservoir level.

Based on the distribution of the air within the storage formation with a maximum gas saturation of $80 \%$ (Figure 2), an elastic model of the underground before and after the CAES injection is built. The elastic properties of the rock matrix (P-wave velocity $V_{p, m}$, S-wave velocity $V_{s, m}$, density $d_{m}$ and porosity $\Phi$ ) are linked with the physical parameters of the fluid and gas phases based on realistic matrix parameters to derive effective medium parameters.

The effective seismic velocities $V_{p}$ and $V_{s}$ and the bulk density $d_{b}$ of the $100 \%$ brine saturated aquifer before the CAES injection can be described by the following averaging equations (Gassmann 1951):

$$
\begin{aligned}
& d_{b}=(1-\Phi) d_{m}+\Phi d_{b r} \\
& \mu_{b}=\mu_{d}=(1-\Phi) \mu_{m} \\
& K_{b}=K_{d}+\left(1-\left(K_{d} / K_{m}\right)\right)^{2} /\left(\Phi / K_{w}+(1-\Phi) / K_{m}-K_{d} / K_{m}^{2}\right) \\
& K_{d}=(1-\Phi) K_{m} \\
& K_{w}=K_{b r} \\
& V_{p}=\operatorname{sqrt}\left(K_{b} / d_{b}\right) \\
& V_{s}=\operatorname{sqrt}\left(\mu_{b} / d_{b}\right)
\end{aligned}
$$

Where d denotes the density, $K$ the bulk moduli and $\mu$ the shear moduli. The subscripts $b, m, d, b r, g$ and $w$ mean the bulk, rock matrix, dry rock, brine phase, gas phase and wetting phase, respectively. For the multiphase flow case after the CAES injection, equations (8) and (12) are adapted to:

$$
\begin{aligned}
& d_{b}=(1-\Phi) d_{m}+\Phi\left(\left(1-S_{g}\right) d_{b r}+S_{g} d_{g}\right) \\
& K_{w}=S_{g} K_{g}+\left(1-S_{g}\right) K_{b r}
\end{aligned}
$$

Based on this rock model Figure 3 shows changes of the material parameters due to the gas injection. Overall the changes of the effective medium parameters within the storage formation are quite small with maximum value changes of $-240 \mathrm{~m} / \mathrm{s}$ for $V_{p},+86 \mathrm{~m} / \mathrm{s}$ for $V_{s}$ and $-168 \mathrm{~kg} / \mathrm{m}^{3}$ for $d_{b}$. The small variations of the $\mathrm{S}$-wave velocity are due to density variations only, while the shear modulus remains constant.

Synthetic data of a reflection seismic survey along the transect is computed by solving the $2 \mathrm{D}$ isotropic elastic equations of motion equations (1-3) with a timedomain finite-difference (FD) technique on a Cartesian grid (Virieux 1986; Holberg 1987; Levander 1988). The reflection seismic acquisition geometry consists of 500 vertical component geophones located at the surface. For the synthetic dataset 100 shots using a vertical impact 


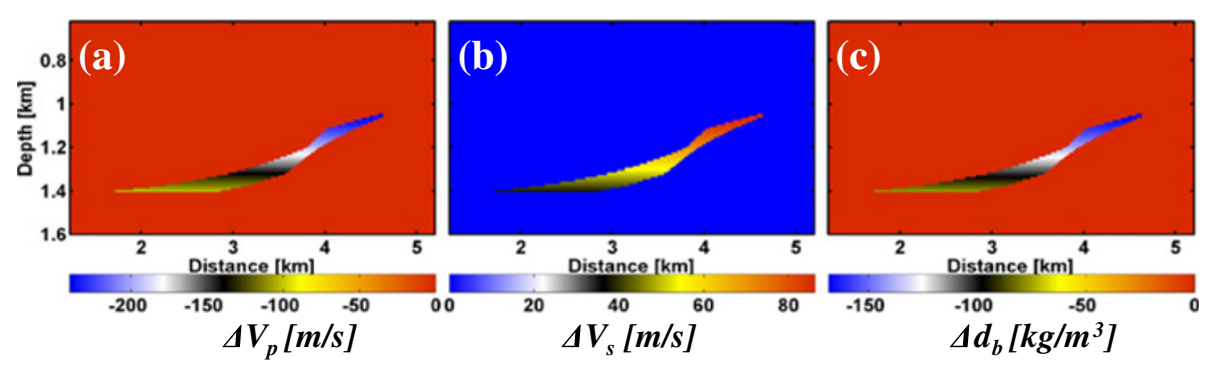

Figure 3 Input (true) changes in elastic parameters due to the gas injection in the Rhaet reservoir below the study site as obtained from the rock model. (a) $\Delta V_{p^{\prime}}$ (b) $\Delta V_{s}$ and (c) density $\left(\Delta d_{b}\right)$.

source are recorded. The source signature is a $20 \mathrm{~Hz}$ Ricker wavelet. Model dimensions along the transect are $6 \mathrm{~km}$ length and $1.8 \mathrm{~km}$ depth. Using an $8^{\text {th }}$ order spatial FD operator, the model can be discretized with $600 \times 180$ grid points with a spatial grid point distance of $10 \mathrm{~m}$. The time is discretized using $\Delta t=1 \mathrm{~ms}$, thus for a recording time of $\mathrm{t}=4 \mathrm{~s}$, approximately 4000 time steps are computed. A free surface boundary condition is assumed on top of the model, while Convolutional Perfectly Matched Layers, CPMLs (Komatitsch and Martin 2007) are used at all other boundaries. The synthetic seismic sections are the input data for the elastic FWI. Figure 4 shows the common shot-gather for shot 50 of the baseline model and the time-lapse data between $t_{1}$ and $t_{0}$ amplified by a factor 10. Notice the strong spurious multiple reflections due to the free boundary condition present in the baseline- and time-lapse data.

To test the robustness of the elastic FWI approach for real field data applications we also investigate the influence of noise and added Gaussian noise within the frequency range of the source signal $(0-40 \mathrm{~Hz})$ with a signal-to-noise ratio $\mathrm{S} / \mathrm{N}=100$ ( $1 \%$ noise) using the SUADDNOISE program of Seismic Unix (Cohen and Stockwell 2008). The resulting shot gathers are shown in Figure 4b,d for the baseline and time-lapse data, respectively.

\section{ERT modelling and parameterization}

At first we introduce briefly the approach for optimized electrode arrays in boreholes applied here. Like surface surveys, ERT data acquisition between two borehole electrode arrays can be conducted in the tripotential quadrupole configurations $\alpha$ (CPPC, $\mathrm{C}=$ current electrode, $\mathrm{P}=$ potential electrode), $\beta(\mathrm{CCPP})$ and $\gamma(\mathrm{CPCP})$. For an $\mathrm{N}$ collinear multi-electrode array, a whole comprehensive data set consists of $[N(N-1)(N-2)(N-3) / 8]$ independent non-reciprocal quadrupole configurations (Noel and Xu 1991). The effective comprehensive data set results from excluding the redundant configurations of less stable inversions from the whole set, i.e., $\gamma$ configurations and those of very large geometric factors (Loke et al. 2010). Resulting comprehensive data set is still
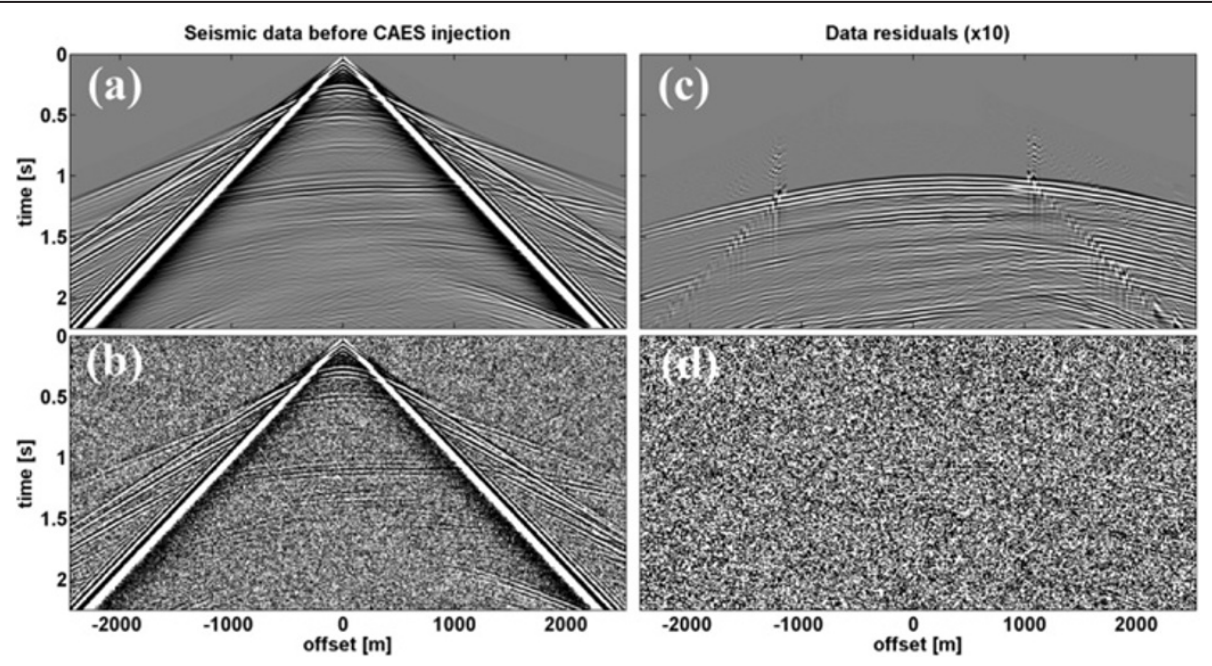

Figure $\mathbf{4}$ Common shot-gather for shot $\mathbf{5 0}$. $(\mathbf{a}, \mathbf{b})$ Baseline model and $(\mathbf{c}, \mathbf{d})$ time-lapse data residual between $\mathrm{t}_{1}$ and $\mathrm{t}_{0}$ amplified by a factor 10. (a, c) Noise-free data and (b, d) noisy data with $\mathrm{S} / \mathrm{N}=100$. 
huge, e.g., a pair of 32 borehole electrodes yield $>10^{6}$ data points. It can map subsurface targets with the highest possible resolution but at very long acquisition times (i.e., poor temporal resolution) and at high costs. Therefore, an optimization approach is based on the model resolution matrix and searches for electrode configurations that maximize the resolution of survey results (e.g., Stummer et al. 2004; Wilkinson et al. 2006). An optimized borehole data sets of practical sizes (15,000 data points) of only $1.5 \%$ of the comprehensive data set but with almost the same spatial resolution is generated in this study (e.g., al Hagrey 2012a). Comparative applications of diverse configurations (standard and nonstandard) show the superiority of the optimized array results (al Hagrey 2012b). Moreover, assessing our optimized array by the technique of region of investigation index (ROI) showed that its inverted tomograms are best constrained by the data coverage in comparison to that of the other configurations (Oldenburg and Li 1999). All these confirm the effectiveness of our optimization approach applied here to generate a practical optimized data set of high resolution.

The 1storage Rhaet formation consists of a highly resistive matrix (e.g., sandstone) and conductive pore brine saturant. The bulk resistivity $(\rho)$ resulting from the gas displacing the brine is predicted using Archie's law (Archie 1942):

$$
\rho=\frac{a \rho_{b r}}{\Phi^{m}\left(1-S_{g}\right)^{n}}
$$

where $\rho_{b r}$ is the brine resistivity, $\Phi$ the porosity, $S_{g}$ the gas saturation and $a, m$ and $n$ are Archie constants. The separate phases (matrix, brine and gas) are assumed without any interaction.

The electrical conductivity of the storage formation is caused mainly by the electrolytes of its pore brine. In the North German Basin, temperature and pressure, and particularly the salinity or total dissolved solids (TDS) increase with depth. Increasing both TDS and temperature causes a dramatic decrease in the resistivity (e.g., Arps 1953; Schlumberger 1985). The TDS rise increases the number of ions carrying electrical currents. The temperature rise increases the salt solubility and decreases the brine viscosity which in turn enhances the ion mobility. The pressure increase with depth, on the other hand, causes a slight increase in the resistivity due to the closure of cracks that are often filled with conductive fluids. However, this effect decreases with increasing depth and is negligible at pressure $>0.3 \mathrm{GPa}$ (e.g., Brace et al. 1965). In formations of the North German Basin, the average vertical gradient (with depth) of brine salinities, temperature and pressure approach $100 \mathrm{mg} / \mathrm{L} / \mathrm{m}, 0.03^{\circ} \mathrm{C} / \mathrm{m}$ and $22.6 \mathrm{kPa} / \mathrm{m}$, respectively (e.g., Magri et al. 2009).
The target layers host borehole electrode arrays at $620 \mathrm{~m}$ offsets within a depth range of $0.9-1.8 \mathrm{~km}$. Each array consists of 32 electrodes at $20 \mathrm{~m}$ spacing. The electric resistivity of the $2 \mathrm{D}$ models are parameterized by the bulk rock resistivity values calculated from Archie equation (17). Here we applied the values of 0.2 for $\Phi, 0.08 \Omega \mathrm{m}$ for $\rho_{b r}$ (corresponding to TDS $\approx 100 \mathrm{~g} / \mathrm{l}$ at $1 \mathrm{~km}$ depth of the North German Basin) and 1, 2 and 2 for constants $a, m$ and $n$ of, respectively, as typical values for the sandstone aquifer. Values of gas saturation are calculated by a potential function simulating their gradual damping with depth.

A 2.5D forward and inverse ERT modelling is carried out using modern codes (RES2DMOD, RES3DMOD $\times$ 64 and RES2DINV $\times 64$ ) based on algorithms by e.g., Loke et al. (2003). The forward modelling code is applied to generate synthetic data sets between each adjacent pair of borehole electrode arrays (including inhole and crosshole) using optimized electrode configurations. These synthetic data sets are generated after gas injection in the brine reservoir of Rhaet formation. The data quality $(0.6 \%$ average simulation error) is confirmed by results of tests on a homogeneous model with a constant $\rho$ value. The technique robustness in the field is realized by adding a random error of commonly $3 \%$ to data sets in addition to their forward simulation error of $0.6 \%$.

In the ERT inversions, diverse setup constraints (mainly regularizations) are applied. These include the minimization methods of least squares $\left(L_{2}\right)$ or robust blocky normalization $\left(L_{1}\right)$, and initial models of a constant homogeneous resistivity or an approximate inverse model (e.g., Claerbout and Muir 1973). Two synthetic data sets (generated before and after adding 3\% random noise) are inverted with incorporating mapping data of subsurface stratigraphy from prior (seismic) surveys (see next sections). Each of these two data sets was inverted twice, once by incorporating layer interfaces and once by fixing resistivity regions, both are outside the reservoir layer.

\section{Gravity modelling and parameterization}

Rock densities depend on the mineral composition, porosity and its content (fluid and gas), pressure $p$, temperature $(T)$, deformations etc. The stratigraphy and average densities applied in the 3D gravity modelling of the study site are based on borehole measurements and data base of Geotectonic Atlas of northwestern Germany (Inselmann 1985; Baldschuhn et al. 2001; Hese 2012). The injected compressed gas in saline reservoirs displaces pore brine and causes a drop of the bulk density which in turn causes a decrease in the gravity components and gradients. The bulk reservoir density $\left(d_{b}\right)$ of partial gas saturations is given by equation (15), whereas dry air density is given by:

$$
d_{g}=p /(R T)
$$


where $R$ is the gas constant $=287.058 \mathrm{~J} /(\mathrm{kgK})$ for dry air. For calculating air and bulk densities (equations 18 and 15), we considered almost realistic values for $p$ (average $27.786 \mathrm{GPa}$ ) and $T$ (average $44^{\circ} \mathrm{C}$ ) prevailing at $1-$ $1.4 \mathrm{~km}$ depth range within the Rhaet reservoir of the North German Basin. This $T$ value results from average local $T$ at sea level $\left(8^{\circ} \mathrm{C}\right)$ plus $T$ fraction caused by the geothermal gradient down to $1.2 \mathrm{~km}$ depth $\left(36^{\circ} \mathrm{C}\right)$. For instance, the average brine and air densities within this depth range approach 1123 and $303 \mathrm{~kg} / \mathrm{m}^{3}$, respectively, and the study sandstone reservoir will suffer from a bulk density drop up to -126 and $-168 \mathrm{~kg} / \mathrm{m}^{3}$ corresponding to air saturations of $S_{g 1}$ for $S_{g 2}$, respectively. Figure 5 shows the 3D distributions of the gas saturation $\left(S_{g 1}\right.$ for $\left.S_{g 2}\right)$ and bulk density (resulting from the parameterization) in the brine reservoir of the anticline flank within the North German Basin.

We used here the software IGMAS+ (Interactive Gravity and Magnetic Application System) designed for 3D gravity, gravity gradient and magnetic modelling (e.g., Götze and Lahmeyer 1988; Schmidt et al. 2011). The model is extrapolated outside the volume of interest in all directions (about two times the model length) to avoid any edge effects. We calculated the gravity field components $\left(g_{z}, g_{y}\right.$ and $\left.g_{z}\right)$ and gradients $\left(g_{z x}, g_{z y}\right.$ and $\left.g_{z z}\right)$ before and after CAES injection, respectively, as well as their difference (residual) anomalies $\left(\Delta g_{x}, \Delta g_{y}\right.$ and $\left.\Delta g_{z}\right)$. Here we show vertical component $\Delta g_{z}$ maps only which reflect the strongest anomalies with respect to CAES reservoirs. We will discuss these gravity anomalies resulting from the two saturations $\left(S_{g 1}\right.$ and $\left.S_{g 2}\right)$ and the lower sensitivity boundary determined for the technique at all saturations, i.e., the least measurable gravity anomaly determined by the modern micro-gravimeter accuracy $(3-5 \mu \mathrm{Gal})$.

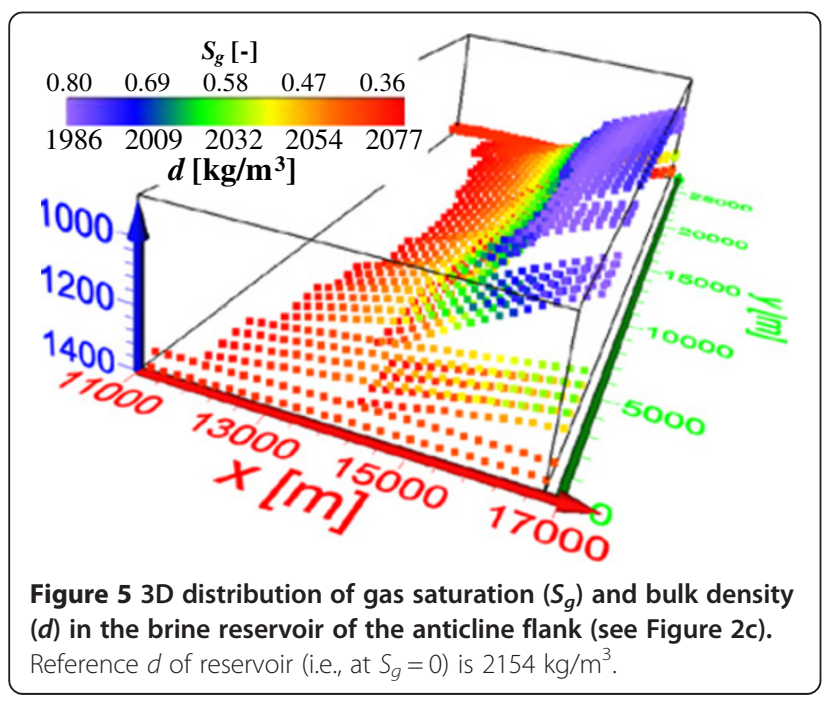

\section{Results of elastic time-lapse FWI}

The initial model for the time-lapse waveform inversion at each time-step is the true elastic medium model before the CAES injection. While this seems to be an overoptimistic assumption, we want to focus this part of the study on the question if the elastic FWI is capable to reconstruct structures at the resolution limit at all. Later we will also investigate the impact of different errors in the baseline model on the elastic time-lapse FWI results. To reduce the nonlinearity of the multiparameter inversion problem a sequential frequency FWI approach is applied. Therefore, Butterworth-lowpass filters are applied to the source wavelet and field data with corner frequencies of 20 and $40 \mathrm{~Hz}$, respectively. To reduce the influence of multiple reflections, exponential time damping (Brossier et al. 2009) of the time-lapse data after the first arrivals are applied. The first arrivals are automatically picked with a STA/LTA picker for the initial model. The detailed elastic FWI workflow is described in Table 1. All material parameters are simultaneously updated.

The inversion results of the noise-free and noisy seismic time-lapse data (Figure 6) can be compared with the true changes of $V_{p}, V_{s}$ and bulk density $d_{b}$ in Figure 3. For the noise-free data (Figure 6, top panel) the shape of the CAES plume, the gradient in the seismic velocities and density could be reconstructed well. The magnitudes of the different material parameters also agree with the true model. The resolution limit of the elastic FWI is roughly one quarter to half of the minimum seismic wavelength. Using a maximum frequency of $60 \mathrm{~Hz}$ with a S-wave velocity of $2074 \mathrm{~m} / \mathrm{s}$ at reservoir level leads to a minimum seismic wavelength of $34 \mathrm{~m}$ and therefore a resolution limit of 9-17 m. This coincides with the FD model discretization errors of $10 \mathrm{~m}$ at the layer interfaces visible in the FWI results. Using the noisy time-lapse data (Figure 6, bottom panel) the resolution of $\Delta V_{p}$ and $\Delta d$ models is also quite good, beside some minor artefacts. Only the $\Delta V_{s}$ model is affected by the Gaussian noise. Further tests (not shown here) with a $\mathrm{S} / \mathrm{N}=50$ ( $2 \%$ noise) lead to even more dominant noise artefacts, but the gas plume is still visible. At $\mathrm{S} / \mathrm{N}=25$ (4\% noise) the gas plume vanishes within the noise artefacts.

\section{Gas quantification by elastic time-lapse FWI}

In most cases gas saturations are estimated from the P-wave velocity of elastic FWI results (Queisser and

\section{Table 1 FWI workflow: corner frequencies $\left(f_{c}\right)$ of Butterworth-lowpass filter for the sequential frequency inversion and time-damping coefficients}

\begin{tabular}{lc}
\hline $\boldsymbol{f}_{\mathbf{c}}[\mathrm{Hz}]$ & Time-damping coefficients $[\mathbf{1 / \mathbf { s }}]$ \\
\hline 20 & $100,50,5,1$ \\
40 & $100,50,5,1$ \\
\hline
\end{tabular}



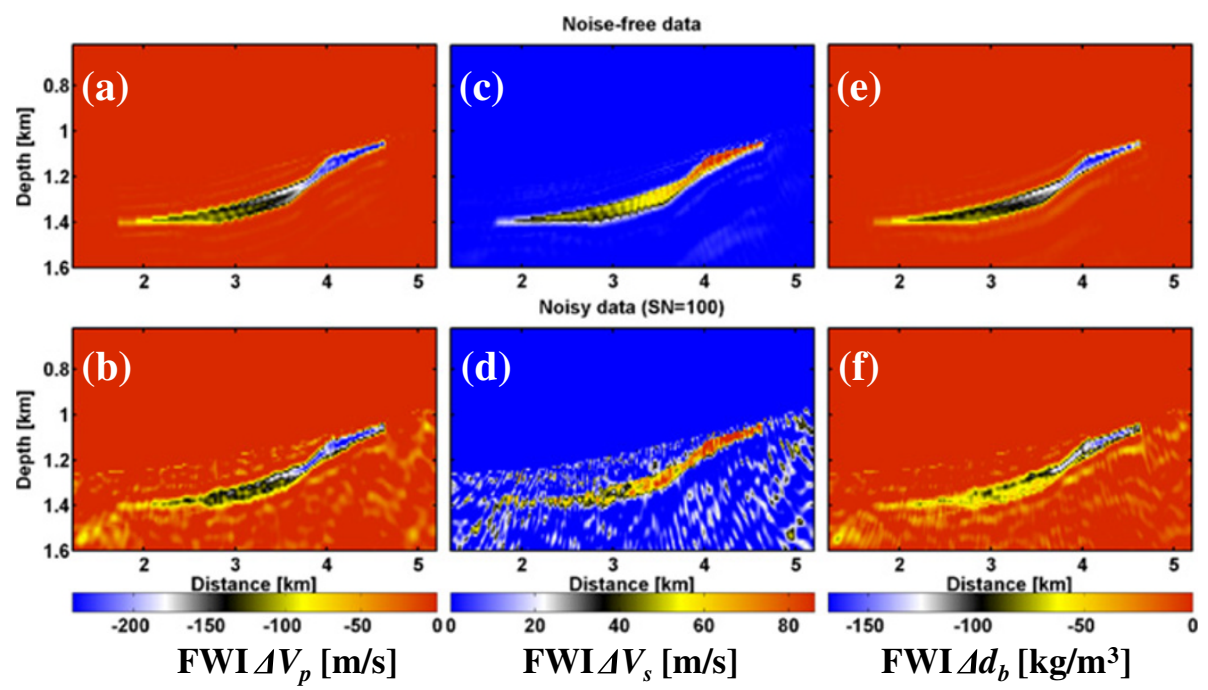

Figure 6 FWI results showing changes in seismic parameters. (a, b) P-wave velocity $\Delta V_{p \text {, }}$ (c, d) S-wave velocity $\Delta V_{s}$ and $(\mathbf{e}, \mathbf{f})$ density $\Delta d$ for the noise-free and noisy dataset $(\mathrm{S} / \mathrm{N}=100)$, respectively (cf., Figure 3 ).

Singh 2012) by inverting the Gassmann equation (10). This involves the estimation of a lot more or less unknown petrophysical parameters. Because the elastic multiparameter FWI delivers a density model, the inversion of equation (15) seems to be more appropriate. By assuming that the brine and gas densities remain constant, the following equation can be derived from equation (15) for the CAES saturation:

$$
\mathrm{S}_{\mathrm{g}}^{\mathrm{FWI}}=\frac{d_{t}-d_{0}}{\Phi\left(d_{g}-d_{b r}\right)}
$$

depending only on the change of the bulk density between time $\mathrm{t}\left(d_{t}\right)$ and the baseline model $\left(d_{0}\right)$, the porosity, as well as brine and gas density. The correct values for porosity and bulk density of the baseline model in the storage formation are used. In Figure 7, the estimated gas saturation changes from the elastic FWI density model (b, c) is compared with the true saturation (a) for the noise-free (b, d) and noisy data (c, e). Using the noise-free data, the extension of the CAES phase and the CAES saturation changes are well recovered. Due to the finite frequency content of the source wavelet, the sharp boundaries of the CAES plume cannot be resolved. Therefore, fictitious large absolute gas saturation errors of about $\pm 10-15 \%$, locally up to $30 \%$, occur at the boundaries (Figure $7 d$, e). The average error within the reservoir approaches about $5 \%$. With the density model from the noisy FWI result

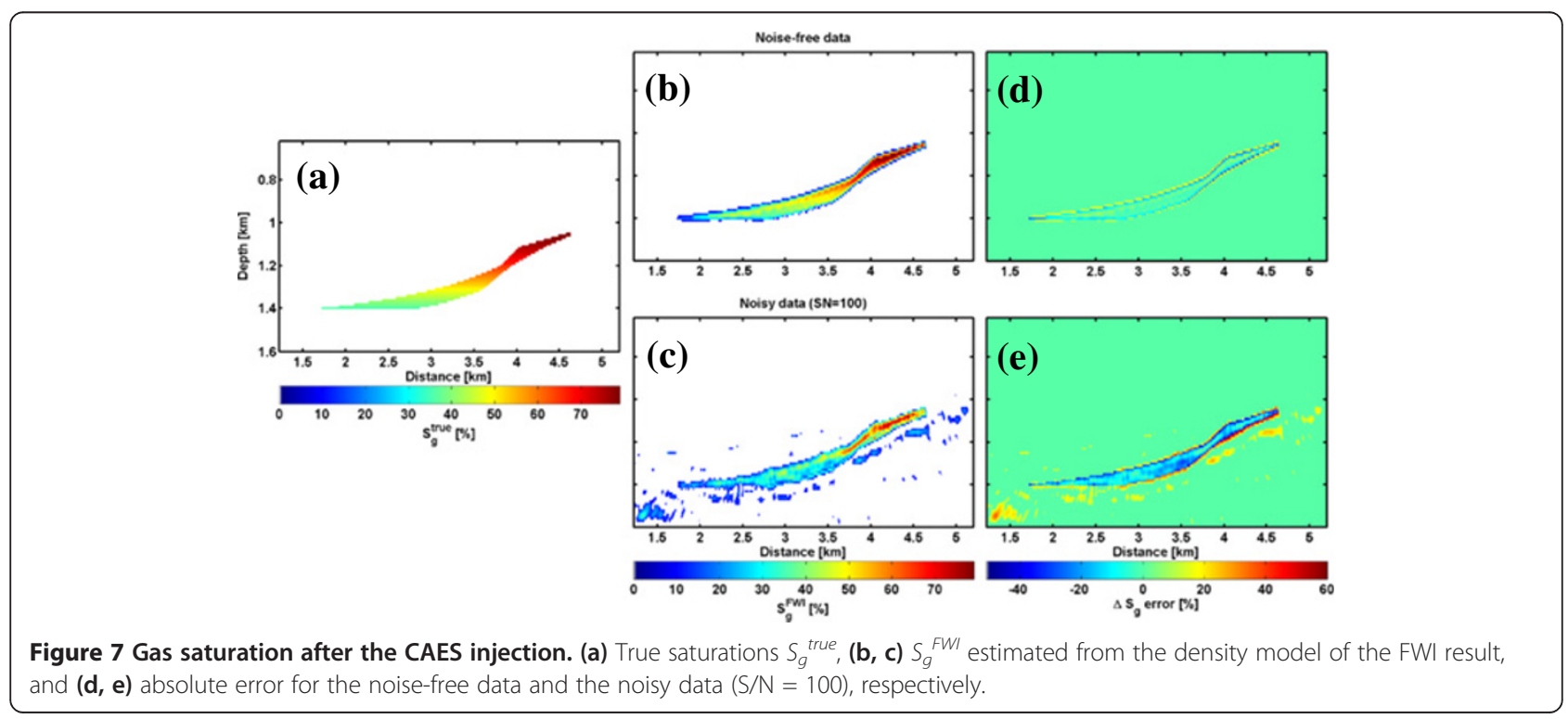


absolute saturation errors increase to $30-60 \%$ at the plume boundaries and $5-30 \%$ within the plume. Noise artefacts outside the plume can lead to errors of up to $20 \%$. For larger S/N-ratios estimated gas saturations become unrealistically larger than $100 \%$.

\section{Impact of errors in the baseline model on the elastic time-lapse FWI results}

So far we only investigated the resolution of elastic timelapse FWI when the baseline model is perfectly known. Figure 8 shows a workflow for the estimation of different baseline models with different kinds of errors. In the first step smooth versions of the true baseline seismic velocity models are generated (Figure 8a), while the density model is assumed to be constant.
With the macro velocity models a pre-stack RTM can be applied to the seismic data to reconstruct the positions of the layer interfaces (Figure 8b). In the next step the interfaces are manually picked (Figure $8 \mathrm{c}$ ) in the zero-offset section and information about the $V_{p}, V_{s}$ and $d_{b}$ model at a borehole location are used to fill the layers with material parameters (Figure 8d, baseline model A). A correct localization of the layer interfaces can be quite complicated, especially in the gas storage layer. As a result velocity and density errors of up to $1000 \mathrm{~m} / \mathrm{s}$ and $300 \mathrm{~kg} / \mathrm{m}^{3}$, respectively, are introduced in the baseline model, even though the medium properties within the layers are lateral homogenous. Beside erroneous multiple reflections within the storage formation due to wrong interface positions, a different diffraction pattern

\section{Estimation of the Baseline Model}

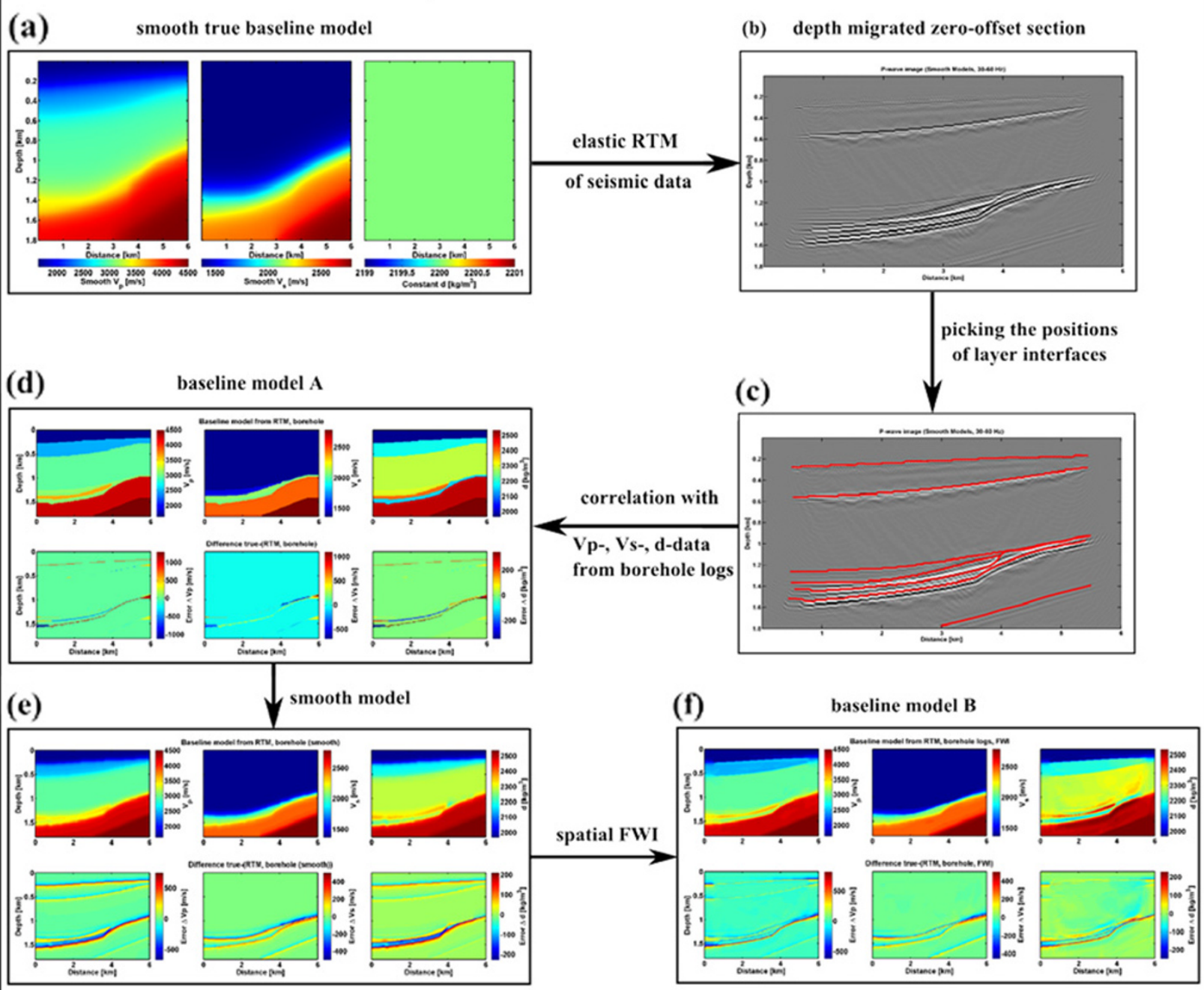

Figure $\mathbf{8}$ Workflow for the estimation of baseline models with different kinds of errors. (a) Smooth true baseline model, (b) depth migrated zero-offset section, (c) layer interfaces, (d) baseline model A, (e) smooth baseline model A, and (f) smooth baseline model B. 
due to changes of model discretization errors on the Cartesian FD-grid become a problem. The elastic time-lapse FWI result for baseline model A is compared in Figure 9d-f with the result for the perfect baseline model (Figure 9a-c). While the position and shape of the gas plume can be reconstructed at least to some extent, the distribution of the material properties within the gas plume are systematically overestimated. To improve the quality of baseline model $\mathrm{A}$, it is smoothed (Figure 8e) with a subsequent application of spatial FWI to the baseline data (Figure 8f, baseline model B). This additional spatial FWI step reduces the errors in the velocity models at the layer interfaces up to a factor two, but the errors are still quite large with $\pm 500 \mathrm{~m} / \mathrm{s}$ for $V_{p}, \pm 400 \mathrm{~m} / \mathrm{s}$ for $V_{s}$, and $\pm 200 \mathrm{~kg} / \mathrm{m}^{3}$ for $d_{b}$. Additionally the smoothing introduces velocity and density errors within the layers. As a result the time-lapse FWI results of baseline model B are only slightly improved (Figure 9g-i) compared to baseline model A (Figure 9d-f).

The evolution of the objective function $E$ during the elastic time-lapse FWI is shown in Figure 10. Transitions between the different inversion stages are defined in Table 1. For the perfect baseline model without noise the objective function significantly decreases during the FWI, while none of such significant decrease is notable when data with a $\mathrm{S} / \mathrm{N}=100$ is inverted. The number of iterations is also reduced. A comparable behaviour occurs for baseline model A and B.

\section{ERT inversion results}

As mentioned before the two synthetic data sets (generated before and after adding 3\% random noise) are inverted with incorporating mapping data of subsurface stratigraphy from prior surveys (e.g. seismic, borehole logs). These two apparent resistivity data sets were inverted by incorporating layer interfaces and fixing resistivity regions, respectively, both are outside the reservoir layer. ERT data inversions reconstruct directly the true subsurface resistivity tomograms including the study gas plume of downward gradual desaturation. This implies no model differencing, unlike seismic results showing the model difference before and after the gas injection. Of all differently independent inversions, every best-fitting tomogram shows least root mean square (rms)-errors of $<0.5 \%$ and iteration number almost of 5 , and is optimized with the $L_{1}$ norm for sharp interfaces. Also this low rms-error value is explained by the good convergence of the synthetic data sets toward the final solution. This $L_{1}$ norm yields significantly more accurate results than $L_{2}$ norm, where the actual subsurface resistivity changes abruptly at sharp target boundaries (cf. Loke et al. 2013). It is more likely to suppose that considerable subsurface information is already available during monitoring from the detailed baseline survey and any other subsequent survey. The a priori incorporation of this mapping data in ERT inversions minimizes the ambiguity of the solution and enhances the resolution of results. Here each data set inversion is constrained by incorporating resistivity regions and interfaces outside the reservoir, respectively.
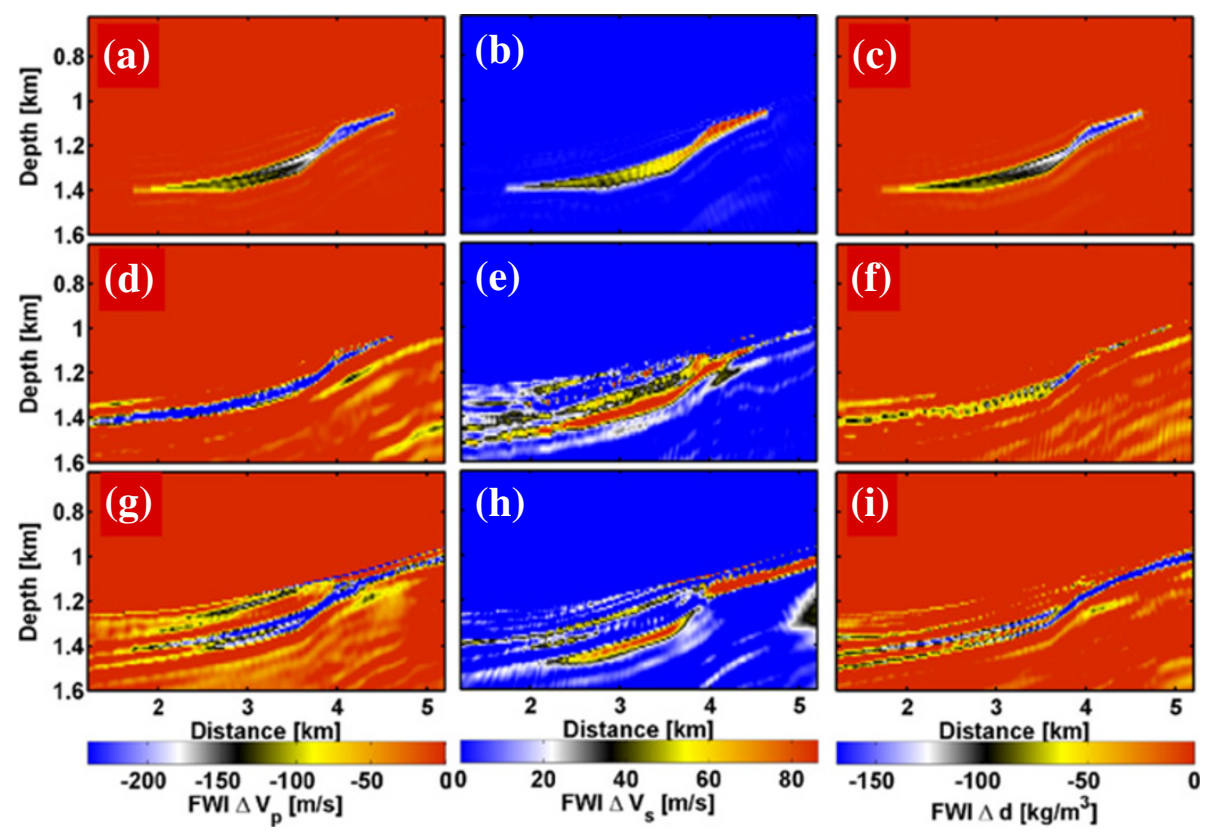

Figure 9 Influence of different baseline models on the elastic time-lapse FWI results. (a-c) Changes of Vp, Vs and density for the perfect baseline model, (d-f) baseline model A estimated from RTM results and borehole logs, and (g-i) baseline model B estimated from RTM results, borehole logs and spatial FWI. 


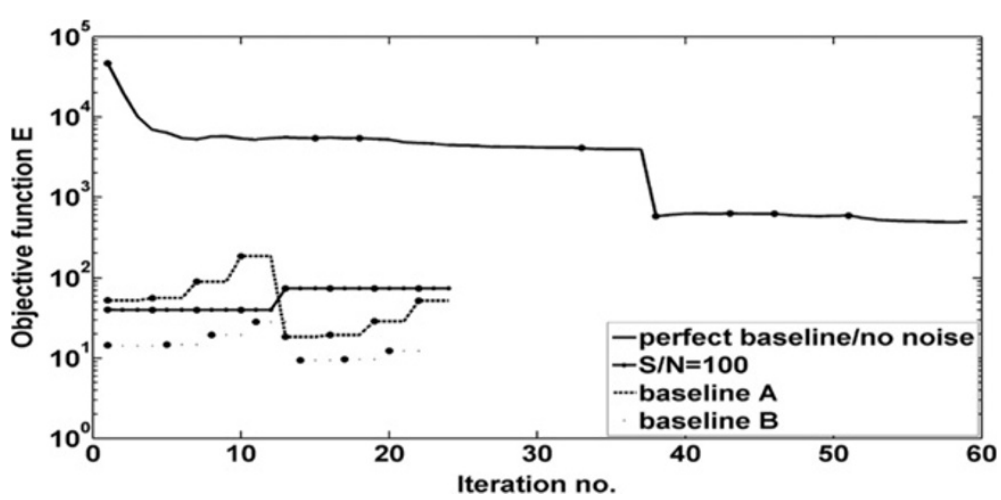

Figure 10 Development of the objective function $E$ (equation 1) during the time-lapse FWI for the perfect baseline/noise free case, inversion with $S / N=100$, baseline model $A$ and baseline model $B$. The solid circles define the transitions between the different inversion stages defined in Table 1.

Moreover, we calculated the model resistivity difference (residual) relative to the input (true) model to quantitatively evaluate the reliability of the reconstructed tomograms. This residual anomaly is a measure for the reliability of ERT inversions. It expresses quantitatively the resolution of the applied technique for both of the spatial mapping capability and the recovering resistivity amplitude. An accurate resistivity amplitude is essential for precise quantifying the injected gas phase saturation using Archie equation (17), see next section. This residual $(\Delta \rho)$ between the corresponding pixels of the input or true $\left(\rho_{\text {true }}\right)$ and output $\left(\rho_{E R T}\right)$ 2D model is calculated by:

$$
\Delta \rho_{r}=\frac{\rho_{\text {true }}-\rho_{\text {ERT }}}{\rho_{\text {true }}} \%
$$

Figure 11 shows only the best-fitting tomograms reconstructed together with their corresponding residuals for the four cases of applying synthetic data before and

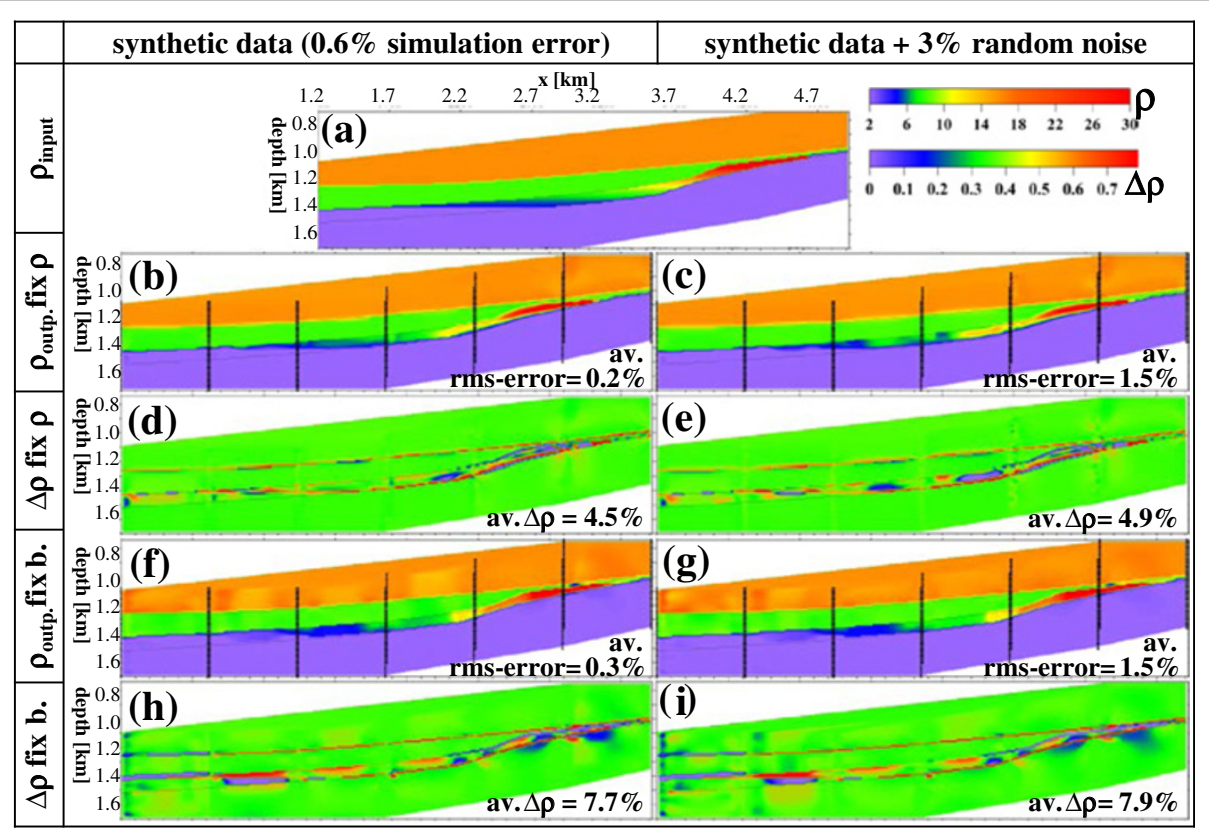

Figure 11 ERT modelling results. (a) Input or true $\left(\rho_{\text {true }}\right)$ models, $(\mathbf{b}, \mathbf{c}, \mathbf{f}, \mathbf{g})$ output resistivity $\left(\rho_{\text {ERT }}\right)$ tomograms with $r m s-e r r o r$, and $(\mathbf{d}, \mathbf{e}, \mathbf{h}, \mathbf{i})$ corresponding residual $\Delta \rho$ models with their average values. Inversion is conducted using constrains of incorporating $\rho$ region (b-e) and boundaries (f-i). These inversions are conducted for synthetic borehole ERT data (contaminated with $0.6 \%$ simulation errors) before and after adding $3 \%$ random noise error. The low residuals (average $<9 \%$ ) reflect well the advantage of constraining of ERT inversions by the seismic mapping results for an accurate reconstruction of the gas phase of downward gradual desaturation. The vertical black dots mark the borehole electrodes and the continuous lines mark the interfaces. 
after adding 3\% random noise errors, and constrained inversions of incorporating $\rho$ regions and boundaries outside the reservoir layer.

Obviously, the ERT inversion results of reconstructed tomograms visualize well the storage targets, particularly the gas plume of a downward gradual desaturation within the brine reservoir for all studied four cases (Figure 11). It is clear that constrained inversion models incorporating resistivity regions are better resolved than these incorporating boundaries. Also the addition of $3 \%$ random error to the synthetic data sets increases the misfit of rms-error values (between input and output response) by a factor of 5-8 but slightly decreases the mapping resolution as reflected by low residual $\Delta \rho$ rise from 4.5 and $7.8 \%$ to 4.9 and $7.9 \%$, respectively. Loke et al. (2013) found that the model inverted with the $L_{1}$-norm is less sensitive to random noise compared with the $L_{2}$-norm.

Resulting residual $\Delta \rho_{r}$ tomograms as a measure for resolution confirm generally the good mapping capability of the applied constrained ERT technique, where all average $\Delta \rho_{r}$ have low values of $4.5-7.9 \%$ with minor deviations (Figure 11). This $\Delta \rho_{r}$ distribution shows that the resulting model reliability (inverse of $\Delta \rho_{r}$ ) is least for the noisy data set inverted by incorporating boundaries and best for the data set without adding random noise inverted with fixing $\rho$ regions. An accurate investigation of $\Delta \rho_{r}$ tomograms show that the resolution suppression due to the addition of $3 \%$ random errors to the generated data (average $\Delta \rho_{r}$ increases from 4.5 to 4.9 for fixing $\rho$ region, and from 7.7 to 7.9 for incorporating boundaries) is lower than that resulting from incorporating boundaries instead of fixing $\rho$ regions (average declines from 4.5 to 7.7 for data without random noise, and from 4.9 to $7.9 \%$ for noisy data).

Constrained inversion tomograms (using any available subsurface data as an a priori information in the ERT inversion) show better resolution (inverse of $\Delta \rho_{r}$. Figure 11) than their corresponding unconstrained or even partly constrained inversion tomograms (not shown here, see al Hagrey et al. 2013). However, these residual $\Delta \rho$ maps still reflect the common smearing effects and artifacts of varying degrees of the ERT technique. This negative effect is particularly visible within the thin storage formation $\left(\Delta \rho_{r}\right.$ up to $\pm 20 \%$ ) with spatially varying gas saturation (i.e., resistivity amplitude). On the other hand the non-varying (homogeneous) formations above (cap rock) and below (aquitard) the storage formation show a $\Delta \rho_{r}$ of almost \pm 0 . Obviously almost all inversion uncertainties are related to the monitored gas phase within the host reservoir. Thus they deliver an error estimate of the injected gas quantities monitored by this constrained inversion technique.

In conclusion, the ERT technique with permanently installed borehole electrodes aims at mapping, monitoring and quantifying the gas volume injected into the saline aquifer at any time. Obviously, the resulting tomograms (Figure 11) fulfil well the spatial mapping and monitoring purposes. This permanent electrode installation helps to maximize the reliability of monitoring data. Modelled anomalies minimizes the background effect and thus maximizes the time-varying response caused here by the injected gas quantity. The residuals (Figure 11) assess and prove the high reliability of the results including the quantification capability for the resistivity amplitude. These highly reliable resistivity amplitudes motivated us to derive the gas saturations (see next section).

\section{Gas quantification by ERT}

The gas phase saturation $S_{g}$ or $S_{g}^{E R T}$ in a partially saturated reservoir medium is driven indirectly by applying the resistivity amplitudes recovered from ERT models (Figure 11) in the Archie equation rearranged as:

$$
S_{g}^{E R T}=1-\left(\frac{a \rho_{b r}}{\rho \Phi^{m}}\right)^{1 / n}
$$

Here, we assume no interaction between the three reservoir phases (solid matrix, brine and gas). Using this equation, saturation models of gas phase $\left(S_{g}{ }^{E R T}\right)$ within the storage reservoir are calculated and plotted together with the corresponding input $\left(\right.$ true,$\left.S_{g}^{\text {true }}\right)$ models and their absolute difference $\left(\Delta S_{g}=S_{g}^{\text {true }}-S_{g}^{E R T}\right)$ in Figure 12 for the four applied cases described before. It is clear that the estimated saturation here contains the uncertainty of the other parameters of $a, m, n, \Phi$ and $\rho_{b r}$ included in this equation. Unlike this, the equation of the resistivity index (the resistivity ratio after and before the injection, e.g., Nakatsuka et al. 2010) eliminates parameters $a, m$ and $\Phi$, whereas parameters $\rho_{b r}$ and $n$ are assumed to stay unchanged with time. However, this index equation includes the uncertainties of both pre- and post-injection $\rho$ models unlike our applied equation (21) which includes the uncertainty of the post injection model only. In the porous sandstone of the Rhaet reservoir, we considered the common values of 1,2 and 2 for the constants $a, m$ and $n$, respectively (Archie 1942), 0.2 for $\Phi$, and $0.08 \Omega \mathrm{m}$ for $\rho_{b r}$ (as mentioned before).

An investigation of resulting models shows that the gradual gas desaturations $\left(S_{g}^{E R T}\right)$ with depth are well mapped within the thin storage formation. The results in Figure 12 show a satisfactory similarity between input (true) and corresponding output (reconstructed) models. The average difference $\Delta S_{g}$ relative to $S_{g}{ }^{\text {input }}$ (Figure 12) approaches $15-21 \%$ for all studied four cases of Figure 11. This small difference confirms again the satisfactory reliability of the results. The gas distribution within the Rhaet reservoir formation shows a good similarity particularly in 


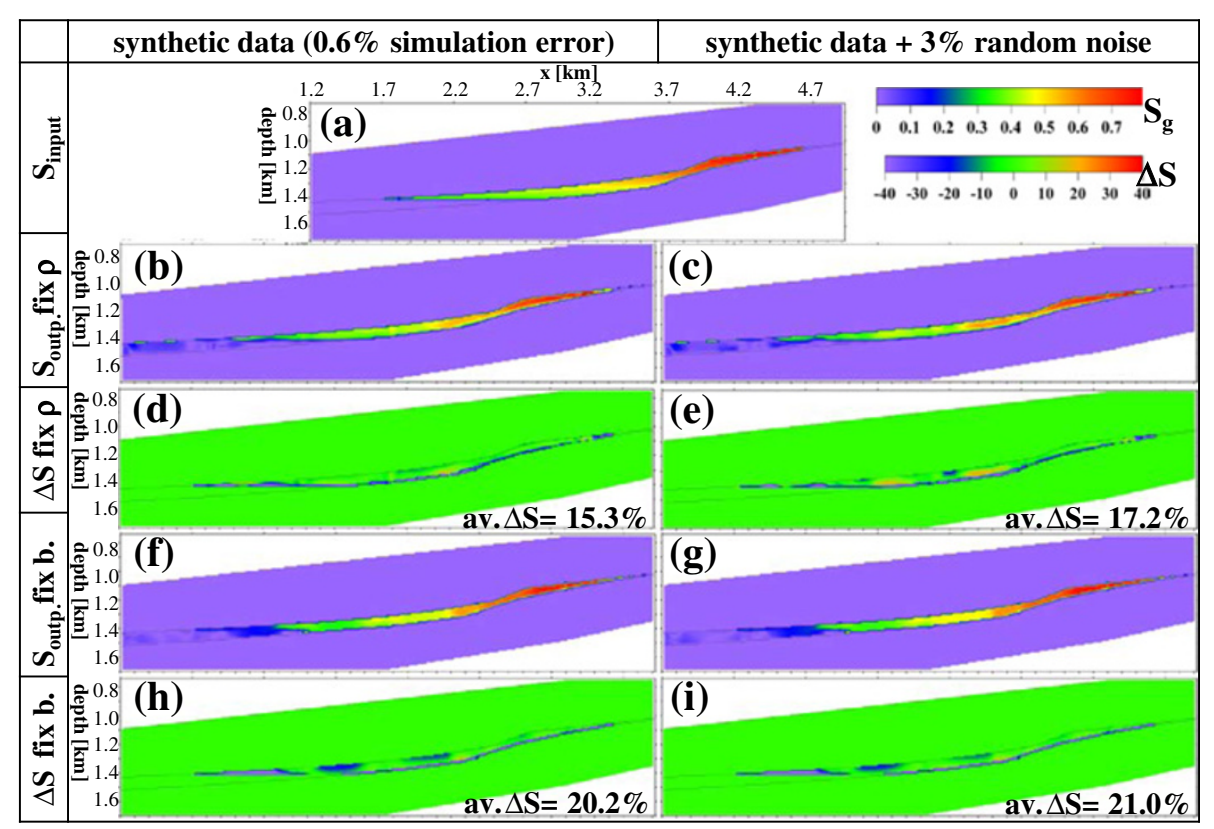

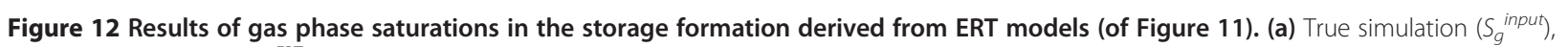
$(\mathbf{b}, \mathbf{c}, \mathbf{f}, \mathbf{g})$ saturations $\left(S_{g}^{E R T}\right)$, and $(\mathbf{d}, \mathbf{e}, \mathbf{h}, \mathbf{i})$ corresponding differences $\left(\Delta S_{g}\right)$ with their average values. The gas phase is recovered satisfactorily everywhere as reflected by $\Delta S_{g}$ values $(\leq 21 \%)$. The continuous lines mark reservoir boundaries.

the region $1-1.4 \mathrm{~km}$ (corresponding to the real distribution) and is insignificant outside this region where its amplitude is below the average $\left(\Delta S_{g}\right)$. Most recovered $S_{g}^{E R T}$ values are lower than their corresponding input ones. This may be related to the smearing effects of the technique. This smearing influences negatively the modelled resistivity amplitude and reduces the resistivity high of the gas plume sandwiched between the two resistivity lows of caprock and aquitard, respectively. High $\Delta S_{g}$ values are concentrated mainly at reservoir interfaces and may be related to discretization errors. At the Cranfield site, Mississipi, Carrigan et al. (2013) found that $\mathrm{CO}_{2}$ saturations measured in monitoring wells are higher than the ERT-derived saturations although both show good spatial correlations. They added that ERT provides an integrated response from large volume, whereas gas sensors ( $\mathrm{dm}$ penetration) provide point measurements and are sensitive to conditions near the well.

\section{D gravity modelling results}

Results of the 3D gravity modelling technique using IGMAS+ program show a high sensitivity to the applied plume scenarios of gas phase injected in the pore Rhaet reservoir of the North German Basin (Figure 13). The negative anomaly amplitude of the vertical gravity component after subtraction the background before gas injections $\left(\Delta g_{z}\right)$ increases with increasing the gas saturation $S_{g}$ causing the mass deficit. The two saturations of $S_{g 1}$
$(60-27 \%)$ and $S_{g 2}(80-36 \%)$, gradually decreasing with depth, show negative anomalies $\Delta g_{z 1}$ and $\Delta g_{z 2}$, respectively, of similar shape, i.e., visually both are hardly distinguishable (Figure 13a, b). The absolute $\Delta g_{z 1}$ amplitude for $S_{g 1}(134 \mu \mathrm{Gal})$ is less than that for $S_{g 2}$ for $S_{g 2}(178 \mu \mathrm{Gal})$. The difference in $\Delta g_{z}$ amplitude (i.e., double difference) between both saturations is visualized well in figure (Figure 13c) and is far higher $(>10$ times) than the measurable accuracy of modern microgravimeters $( \pm 3-5 \mu \mathrm{Gal})$. Fewer saturation changes are verified systematically at the whole range of saturations. We found that $1 \%$ change in saturations $\Delta S_{g}$ yield 2.2 $\mu \mathrm{Gal}$ change in $\Delta g_{z}$ for our study gas plume. This implies that the technique can monitor a saturation change $\Delta S_{g}$ down to $2.5-3 \%$ for the whole saturation range. This $\Delta S_{g}$ range results in a $\Delta g_{z}$ changes above 5 $\mu \mathrm{Gal}$. Obviously, time-lapse data do not require many corrections (e.g., free-air, Bouguer, terrain) but temporal shallow changes (e.g. fluctuations of the water table) highly affect the gravity readings. Our gravity modeling of a water table at $10 \mathrm{~m}$ depth below the study site yields a measurable $5 \mu \mathrm{Gal}$ anomaly (micro-gravimeter accuracy) already for $0.5 \mathrm{~m}$ fluctuations only. Therefore, such fluctuations should be observed in wells to remove their effects from gravity readings. In conclusion, the $3 \mathrm{D}$ gravity modelling technique applied here is able to monitor timelapses of gas saturations of $S_{g 1}$ and $S_{g 2}$ as well as any saturation changes $\left(\Delta S_{g}\right)$ down to $\pm 3 \%$ within the whole range of saturation. 


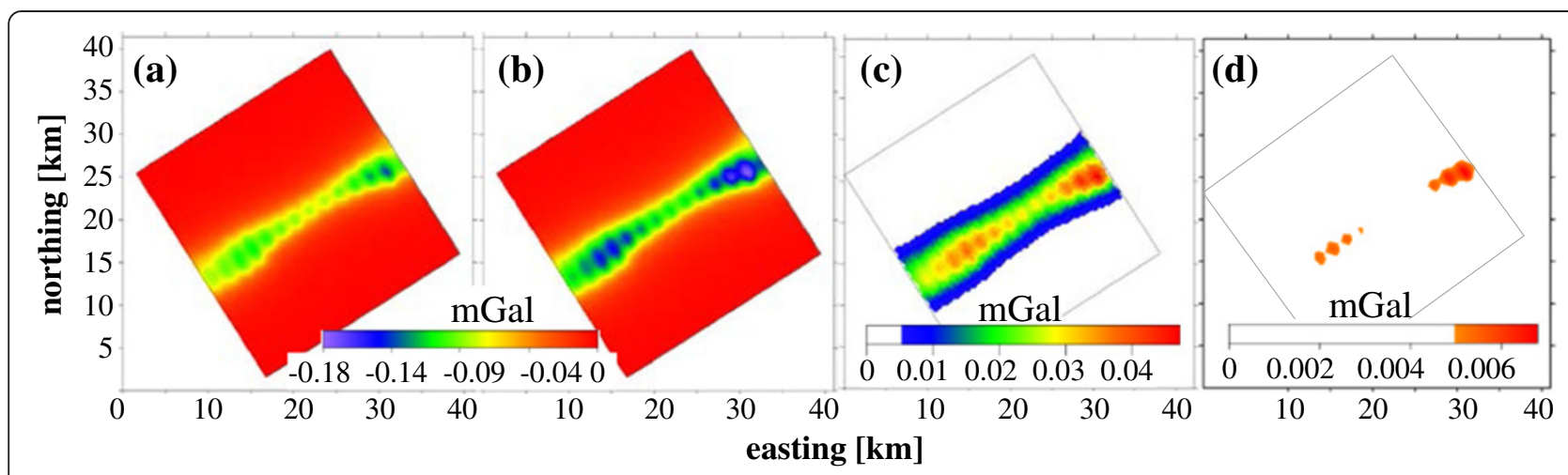

Figure 13 3D forward gravity modelling of gas storages in the anticline limb of Rhaet sandstone formation below the study site.

(a) Vertical gravity anomalies $\Delta g_{z}$ (relative to background) for the gas reservoir with saturations $S_{g 1}$, (b) $\Delta g_{z}$ for $S_{g 21}$ (c) their anomaly difference, and (d) least anomaly difference measurable by micro-gravimeters of $\geq 5 \mu \mathrm{Gal}$ resulting from a saturation change $\Delta S_{g}$ of $\pm 3 \%$. Only the measurable anomaly part is visualized in c-d by blanking colour bar below $5 \mu \mathrm{Gal}$ (immeasurable limit).

\section{Discussion and conclusion}

Mitigation of anthropogenic green house gases demand developments of renewable energy resources. However, most renewable energy sources are intermittent and therefore need buffer storage to match public power supply and demand. In geologic storages, replacing pore brine with compressed gas energy within the reservoir formation causes changes in elastic and electric properties and justify applications of integrative geophysical methods for monitoring this energy storage. We apply here elastic FWI, ERT and gravity techniques to map and quantify a thin gas plume of gradual downward desaturation injected in a deep brine aquifer. These aimed tasks are real challenges for any singly applied geophysical monitoring technique.

In this numerical study we simulate a nearly realistic storage scenario by considering: (1) the study site is chosen inside the North German Basin of favourable conditions for energy storages, (2) a storage model scenario is parameterized by real (published) data for this basin, (3) the gas phase plume is simulated with downward gradual desaturation similar to realistic cases, and (4) a common random noise level is added to the synthetic data to robust the technique for real field applications. The aimed resolution is enhanced by applying: (1) an integrative approach of geophysical methods, (2) an optimized approach for data acquisitions with a data coverage constraining well the inversion model and maximizing the resolution, and (3) constrained inversions to minimize interpretation ambiguities (by a priori use of available data, e.g. seismic, logs).

Unlike classical travel-time based tomographic approaches, the elastic FWI is capable to map the extension of the thin gas plume of downward gradual desaturation using only reflection seismic data, if a very accurate background model for the seismic velocities and density can be estimated before the gas injection.
Additionally the elastic FWI recovers the changes of isotropic elastic material parameters and density due to the gas injection and subsequent partial drainage of the aquifer. By using an appropriate rock model, changes of the gas saturation can be deduced from the elastic FWI results with an accuracy of 5-30\% within the aquifer, depending on the amount of noise $(\mathrm{S} / \mathrm{N}$-ratio $>100)$ present in the data. Due to the finite frequency content of the source signal larger saturation errors up to $60 \%$ can occur at the boundaries of the gas plume. Density inversion artefacts outside the aquifer due to noise can lead to fictitious estimates of saturation variations with local maxima of $20 \%$. For a S/N-ratio of 50 the shape of the gas plume is still visible, but estimations of the gas saturation become highly erroneous and a $\mathrm{S} / \mathrm{N}$-ratio of 25 seem to be the detection limit for the gas plume. Errors in the elastic baseline model has a very substantial impact on the quality of the elastic time-lapse FWI results. Picking errors in layer interfaces and inaccurate material parameters within the layers lead to results with an approximately correct shape and position of the gas plume, but overestimated wrong elastic material parameters within the gas plume. Therefore, the estimation of accurate elastic baseline models for a successful elastic multiparameter FWI, and a subsequent calculation of the gas saturation distribution, is the greatest challenge for real field applications. Smooth macro-velocity models based on Common-Reflection Surface (CRS) stacking (Mann 2002) and Normal-Incidence Points (NIP)-wave tomography (Duveneck 2004) for P- and SH-wave data combined with the intensive use of prior information from borehole logs seem to be the most promising approach.

The applied constrained ERT inversion technique (taking use from previous seismic mapping and well logs) is also able to accurately map storage targets (caprock, reservoir with thin gas and aquitard) in all four applied cases (resulting from inverting noise-free and noisy data 
by incorporating layer boundaries and resistivity regions, respectively). The technique can even recover the gas plume of downward gradual desaturation with a good resolution. Also inversion models constrained by incorporating resistivity regions are better resolved than these constrained by incorporating boundaries, both are applied outside the reservoir layer. The thin resistive gas plume is sandwiched between the two conductive layers of the overlying caprock and the underlying aquitard. Based on the equivalence principle, the resistance $(\rho * h$, $\rho=$ resistivity, $h=$ thickness) of this thin resistive plume can hardly be resolved into $\rho$ and $h$. This normally results in smearing with blurred boundaries and larger volume relative to the input model. These common ERT limitations are minimized here by applying the constrained inversion approach taking use of any available subsurface data. Uncertainties in mapping structures and quantifying the resistivity amplitudes are relatively low reflecting the high reliability of the reconstructed results.

Notably we could quantify reliably the gas saturations indirectly from the density and resistivity models resulting from the inversion by applying common petrophysical equations. The saturation results deduced from ERT technique fit well their corresponding values derived from elastic FWI. Both show reasonable absolute average differences $(<20 \%)$ relative to the background. However, such results should be cautiously treated, where their validity and uncertainty should be studied in real field data.

The use of synthetic data contaminated with random error may reflect the real world of data. Obviously, adding random noise (typically 3\%) to the synthetic ERT data increases the rms-error values by a factor of 5-9 but slightly decreases the mapping resolution. Our results here are in accordance with that obtained by al Hagrey (2012a) for ERT applications in CCS modelling. Using modelling codes (as applied here) and adding a random noise in an ascending order (1,2, and 5\% levels) to the synthetic data sets generally increases the rmserrors by a factor of 2 to 9 but slightly decreases the mapping capability of ERT technique. Ramirez et al. (2005) obtained similar results and concluded that the effect of the random error in ERT is insignificant for anomalies of a large size and magnitude.

Obviously the elastic FWI and ERT modelling using $2.5 \mathrm{D}$ codes has been conducted along a $2 \mathrm{D}$ section of the geological 3D model applied in the modelling simulation. This 2D model simplification is fully justified by the evidence that this $2 \mathrm{D}$ section cuts the main (storage) structure (gentle anticline within almost horizontal layering) along its main strike.

In conclusion results reveal the capability of our applied integrative geophysical approach to resolve the
CAES targets and to quantify intrinsic property changes of the injected gas saturation in the reservoir. Constrained inversion models of elastic FWI and ERT are even able to recover well the gradual desaturation with depth. The accurately mapped spatial (seismic and electric) parameters are applied in their respective petrophysical equation to yield precise quantifications of gas saturations from each technique independently. Both resulting saturation models are in accordance with each other and with the input (true) saturation model. A joint elastic FWI and ERT inversion has a high potential to improve the applicability of the approach (e.g. Karaoulis et al. 2012). In a numerical study of a moving gas front within a reservoir, the joint inversion of seismic and electric time-lapse data sets reduces the presence of artifacts, and can retrieve the shape and estimate parameter better than their individual (unconstrained) inversions. For time-lapse data ERT uses permanently installed borehole electrodes, whereas seismic data needs to be repeated such that the source and receiver positions could not be the same and therefore more uncertainties can occur.

Moreover, the applied 3D gravity technique shows high sensitivity to the mass deficit resulting from the storage of the gas phase. The vertical gravity component can resolve saturations and saturation changes down to $\pm 3 \%$ assuming that the data is corrected for temporal fluctuation effects of the groundwater table.

\section{Competing interests}

The authors declare that they have no competing interests.

\section{Authors' contributions}

The authors, viz SAH, DK and WR with the consultation of each other carried out this work and drafted the manuscript together. All authors read and approved the final manuscript.

\section{Acknowledgement}

We thank D. De Nil for fruitful discussions, D. Wehner, M. Merz, B. Weise for computer work, the Editor and three anonymous reviewers for their constructive comments to improve the paper. Special thanks go to H.-J. Götze and S. Schmidt for supplying the license of the software IGMAS+ used for gravity modelling. This study has been carried out within the framework of research project "ANGUS+" funded by the German Federal Ministry of Education and Research (BMBF).

Received: 11 January 2014 Accepted: 30 April 2014

Published: 25 May 2014

\section{References}

al Hagrey SA (2012a) 2D optimized electrode arrays for borehole resistivity tomography and $\mathrm{CO}_{2}$ sequestration modelling. Pure and Applied 169(7):1283-1292

al Hagrey SA (2012b) 2D model study of $\mathrm{CO}_{2}$ plumes in saline reservoirs by borehole resistivity tomography. International Journal of Geophysics Article ID 805059:12, doi:10.1155/2011/805059

al Hagrey SA, Strahser M, Rabbel W (2013) Seismic and geoelectric modelling studies of parameters controlling $\mathrm{CO}_{2}$ geostorage in saline reservoirs. International Journal of Greenhouse Gas Control 19:796-806

Archie GE (1942) The electrical resistivity log as an aid in determining some reservoir characteristics. Transactions of the American Institute of Mining Engineers 146:54-62 
Arps JJ (1953) The effect of temperature on the density and electrical resistivity of sodium chloride solutions. Transactions of the American Institute of Mining Engineers, AIME 98:327-330

Asnaashari A, Brossier R, Garambois S, Audebert F, Thore P, Virieux J (2013a) Regularized seismic full waveform inversion with prior model information. Geophysics 78(2):R25-R36

Asnaashari A, Brossier R, Garambois S, Audebert F, Virieux J (2013b) Targetoriented time-lapse imaging using FWI with prior model information. In: EAGE Expanded Abstracts, 75th EAGE Conference \& Exhibition, London, We P07 06

Baldschuhn R, Frisch U, Kockel F (2001) Geotektonischer Atlas von Nordwest-Deutschland und dem deutschen Nordsee-Sektor - Strukturen, Strukturentwicklung, Paläogeographie. Geol. Jb, A153, Hannover (BGR)

Bauer S, Beyer C, Dethlefsen F, Dietrich P, Duttmann R, Ebert M, Feeser V, Görke U, Köber R, Kolditz O, Rabbel W, Schanz T, Schäfer D, Würdemann H, Dahmke A (2013) Impacts of the use of the geological subsurface for energy storage: an investigation concept. Environmental Earth Sciences 70:3935-3943

Brace WF, Orange AS, Madden TR (1965) The effect of pressure on the electrical resistivity of water-saturated crystalline rocks. J Geophys Res 70(22):5669-5678

Brossier R (2011) Two-dimensional frequency-domain visco-elastic full waveform inversion: parallel algorithms, optimization and performance. Comput Geosci 37:444-455

Brossier R, Operto S, Virieux J (2009) Seismic imaging of complex onshore structures by two-dimensional elastic frequency-domain full-waveform inversion. Geophysics 74(6):WCC63-WCC76

Carrigan CR, Yang X, LaBrecque DJ, Larsen D, Freeman D, Ramirez AL, Daily W Aines R, Newmark R, Friedmann J, Hovorka S (2013) Electrical resistance tomographic monitoring of $\mathrm{CO}_{2}$ movement in deep geologic reservoirs. International Journal of Greenhouse Gas Control 18:401-408

Claerbout JF, Muir F (1973) Robust modeling with erratic data. Geophysics 38:826-844

Cohen JK, Stockwell JJW (2008) CWP/SU: seismic unix release No. 41: an open source software package for seismic research and processing. Center for Wave Phenomena, Colorado School of Mines

Crotogino F, Mohmeyer K-U, Scharf R (2001) Huntorf CAES: more than 20 years of successful operation. In: Solution Mining Research Institute meeting, Orlando, Florida, USA

Denli H, Huang L (2009) Double-difference elastic waveform tomography in the time domain, SEG technical program expanded abstracts., pp 2302-2306

Duveneck E (2004) Tomographic determination of seismic velocity models with kinematic wavefield attributes. Ph.D. thesis, Karlsruhe University, Logos Verlag Berlin

Gassmann F (1951) Über die Elastizität poröser Medien. Vierteljahrsschrift der Naturforschenden Gesellschaft in Zürich 96:1-23

Götze H-J, Lahmeyer B (1988) Application of three-dimensional interactive modeling in gravity and magnetics. Geophysics 53(8):1096-1108

Graupner BJ, Li D, Bauer S (2011) The coupled simulator ECLIPSE - OpenGeoSys for the simulation of $\mathrm{CO} 2$ storage in saline formations. Energy Procedia 4:3794-3800

Hese F (2012) 3D Modellierungen und Visualisierung von Untergrundstrukturen für die Nutzung des unterirdischen Raumes in Schleswig-Holstein. Ph.D. thesis, University of Kiel, Kiel, p 156

Holberg O (1987) Computational aspects of the choice of operator and sampling interval for numerical differentiation in lage-scale simulation of wave phenomena. Geophys Prospect 35:629-655

IEA (International Energy Agency) (2013) World energy outlook special report 2013: redrawing the energy climate map. OECD/IEA, Paris

Inselmann R (1985) Dichte-Messungen in der Bundesrepublik Deutschland. Erdöl-Erdgas 101(3):76-78

Karaoulis M, Revil A, Zhang J, Werkema DD (2012) Time-lapse joint inversion of crosswell DC resistivity and seismic data: a numerical investigation. Geophysics 77:D141-D157

Köhn D, De Nil D, Kurzmann A, Przebindowska A, Bohlen T (2012) On the influence of model parametrization in elastic full waveform tomography. Geophys J Int 191:325-345

Komatitsch D, Martin R (2007) An unsplit convolutional perfectly matched layer improved at grazing incidence for the seismic wave equation. Geophysics 72 (5):SM155-SM167

Landau LD, Lifschitz EM (1986) Theory of elasticity. Elsevier

Levander A (1988) Fourth order finite-difference P-SV seismograms. Geophysics 53(11):1425-1436
Loke H, Acworth I, Dahlin T (2003) A comparison of smooth and blocky inversion methods in 2D electrical imaging surveys. Explor Geophys 34:182-187

Loke MH, Wilkinson PB, Chambers JE (2010) Fast computation of optimized electrode arrays for 2D resistivity surveys. Comput Geosci 36:1414-1426

Loke MH, Dahlin T, Rucker DF (2013) Smoothness-constrained time-lapse inversion of data from 3D resistivity surveys. Near Surface 11:20, doi:10.3997/ 1873-0604.2013025

Magri F, Bayer U, Pekdeger A, Otto R, Thomsen C, Maiwald U (2009) Salty groundwater flow in the shallow and deep aquifer systems of the SchleswigHolstein area (North German Basin). Tectonophysics 470(1-2):183-194

Makhijani A, Mills C, Ramana MV (2012) Renewable Minnesota: a technical and economic analysis of a $100 \%$ renewable-energy based electricity system for Minnesota., Institute for Energy and Environmental Research (IEER), http:// ieer.org/resource/reports/renewable-minnesota-technical

Mann J (2002) Extensions and applications of the common-reflection-surface stack method. Ph.D. Thesis, Karlsruhe University, Logos Verlag Berlin

Mora P (1987) Nonlinear two-dimensional elastic inversion of multi offset seismic data. Geophysics 52:1211-1228

Nakatsuka Y, Xue Z, Garcia H, Matsuoka T (2010) Experimental study on $\mathrm{CO}_{2}$ monitoring and quantification of stored $\mathrm{CO}_{2}$ in saline formations using resistivity measurements. Int J Greenh Gas Con 4:209-216

Nocedal J, Wright SJ (2006) Numerical optimization. Springer, New York

Noel M, Xu B (1991) Archaeological investigation by electrical resistivity tomography: a preliminary study. Geophys J Int 107:95-102

Oldenburg DW, Li Y (1999) Estimating depth of investigation in dc resistivity and IP surveys. Geophysics 64:403-416

Queisser M, Singh SC (2012) Full waveform inversion in the time lapse mode applied to $\mathrm{CO}_{2}$ storage at Sleipner. Geophys Prospect 61:537-555

Ramirez AL, Nitao JJ, Hanley WG, Aines R, Glaser RE, Sengupta SK, Dyer KM, Hickling TL, Daily WD (2005) Stochastic inversion of electrical resistivity changes using a Markov Chain Monte Carlo approach. J Geophys Res 110:18, B02101, doi:10.1029/2004JB003449

Schlumberger (1985) Schlumberger log interpretation charts, Schlumberger Well Services. Schlumberger Limited, New York

Schmidt S, Plonka C, Götze H-J, Lahmeyer B (2011) Hybrid modelling of gravity, gravity gradients and magnetic fields. Geophys Prospect 59(6):1046-1051

Shipp RM, Singh SC (2002) Two-dimensional full wavefield inversion of wideaperture marine seismic streamer data. Geophys J Int 151:325-344

Stummer P, Maurer H, Green AG (2004) Experimental design: electrical resistivity data sets that provide optimum subsurface information. Geophysics 69:120-139

Succar S, Williams RH (2008) Compressed air energy storage: theory, resources, and applications for wind power. Princeton Environmental Institute Princeton University, Princeton, p 81

Tarantola A (1986) A strategy for non-linear inversion of seismic reflection data. Geophysics 51(10):1893-1903

Virieux J (1986) P-SV wave propagation in heterogeneous media: velocity-stress finite-difference method. Geophysics 51(4):889-901

Virieux J, Operto S (2009) An overview of full waveform inversion in exploration geophysics. Geophysics 74(6):WCC127-WCC152

Wilkinson PB, Meldrum PI, Chambers JE, Kuras O, Ogilvy RD (2006) Improved strategies for the automatic selection of optimised sets of electrical resistivity tomography measurement configurations. Geophys J Int 167:1119-1126

\section{doi:10.1186/2193-1801-3-267}

Cite this article as: al Hagrey et al:: Geophysical assessments of renewable gas energy compressed in geologic pore storage reservoirs. SpringerPlus 2014 3:267. 